\title{
DETECTION, CLASSIFICATION AND ANALYSIS OF EVENTS IN TURBULENCE TIME SERIES
}

\author{
YANFEI KANG
}

(Received 6 January 2015; first published online 26 February 2015)

\section{Mathematics subject classification: primary 76F55; secondary 62M10. \\ Keywords and phrases: atmospheric turbulence, time series, noise.}

Time series are the major source of information to study characteristics of the atmospheric boundary layer (ABL), which is frequently dominated by various types of events embedded in the time series with different levels of noise. To understand physical dynamics of the atmospheric turbulence, the individual events need to be detected and studied for their physical and structural characteristics. In spite of the attention that has been given to studying events among the atmospheric science community, the detection of events still presents a challenge and thus their characteristics and contributions to the ABL remain poorly understood. Besides the existence of high-level noise in turbulence, the main difficulty is that many of the events that are responsible for the variability in the atmospheric turbulence time series are previously unknown, especially in the stable ABL.

This dissertation develops a new method for detecting and classifying structures from turbulence time series. The main idea of the method is in defining events as timeseries subsequences that are significantly different from noise. This switches the focus of the event-detection approach towards defining the characteristics of noise, which is in many situations an easier problem than defining a structure. For atmospheric time series, a natural characterisation of the noise is red noise, which is a stationary AR(1) process. The proposed method consists of two steps. The first step of the method is event extraction based on noise tests. We perform a noise test on each subsequence extracted from the series using a sliding window. All the subsequences recognised as noise are removed from further analysis and the events are extracted from the remaining non-noise subsequences. This step does not assume particular geometries or amplitudes of the flow structures. In the second step, the detected structures are classified into groups with similar characteristics. This step groups large

Thesis submitted to Monash University in May 2014; degree approved 19 August 2014; supervisors: Kate Smith-Miles and Danijel Belušić.

(c) 2015 Australian Mathematical Publishing Association Inc. 0004-9727/2015 \$16.00 
numbers of detected events such that it opens a pathway for the detailed study of their characteristics and helps to gain understanding of events with previously unknown origin. In order to account for the underlying characteristics of the extracted events, a feature-based clustering method is used. This method first summarises each event with its global measures before performing clustering in the feature space. It yields substantially better results than clustering based on raw data of the events.

The developed $R$ package TED is tested on artificial time series with different levels of complexity and real-world atmospheric turbulence time series. The results on artificial data show that events used to generate the data can be exactly detected and clustered. The method is robust to high levels of noise, which is advantageous regarding very noisy turbulence time series. Application of the method to a wellknown real-world turbulence dataset demonstrates that the method successfully extracts realistic flow structures, which are in line with previous studies that have examined the underlying physical mechanisms of several isolated events on that dataset. The proposed method is then applied to a more complicated turbulence dataset about which no published results can be found regarding extraction of unknown events. The proposed method is able to detect and distinguish events with different dynamical characteristics even though the clustering step is based only on statistical measures of characteristics of events from time series.

\section{YANFEI KANG,}

School of Mathematical Sciences, Monash University, Australia e-mail: yanfei.kang@monash.edu 